\title{
Characterization of the sonority associated with woodwinds instruments through spectral analysis
}

\author{
Yubiry González, Ronaldo C. Prati \\ Center of Mathematics, Computer Science and Cognition - CMCC \\ Federal University of ABC - UFABC. Av. Dos Estados, 5001, Bangú \\ Santo André, 09210-580, SP, Brazil \\ Yubiry.gonzalez.17@gmail.com,rcprati@gmail.com
}

\section{Extended Abstract}

The sonority is one of the widely used definitions by musicians when trying to define the color or the balance of timbres associated with individual or groups of instruments, such as for ensembles or orchestras. Currently, those who use this term refer to subjective parameters associated with the "balance of tones," "amplitude of sound," and "mixtures of colors," using terms such as bright, opaque, robust, weak, among others, which they do not have a precise definition. Other theoretical musicians also associate sonority with relations between consonances, dissonances, and other harmonic aspects that may exist in musical compositions [1]. Other trends associated with electroacoustic music use the new synthesized sound resources to achieve mixtures of new colors, or sonority other than those obtained only with acoustic instruments.

The perception of sound is characterized by amplitude, tone, and timbre mainly, being related to physical-acoustic parameters, such as intensity, frequency, and the number of harmonics, respectively. Furthermore, it is well known that the number and relative intensity of the harmonics or secondary frequencies, corresponding to an individual sound, vary according to the type of instrument and its details in the manufacture (geometry and materials) [2]. In the same way, but the second-order, there is the corresponding to the own musical performance carried out by each interpreter which can originate, under the same acoustic conditions and the same instrument, different sonorities.
From the use of digital signal processing tools, these secondary or harmonic frequencies can be analyzed, allowing a direct gathering of information about the timbre or colors associated with each instrument. This process is useful for the characterization of the sonority due to its high relation with the timbre. Various studies have been carried out in this direction for woodwind instruments such as the transverse flute and the clarinet [3] that propose studying the timbre to extract information from the different possible colors in any musical instrument, proposing for its characterization a spectral centroid with the use of digital signal analysis [4].

For the present work samples of audio signals were obtained from three wooden wind instruments, such as transverse flute, oboe, and clarinet, which under optimal acoustic conditions executed melodic patterns of sounds with the same frequency, scales and musical fragments, following the methodology of González, Y. [5]. Then with the obtained audio samples, different spectral analyses were performed using Fast Fourier Transform (FFT), Spectral Power Density (DPE) and Spectrograms. Finally, ten professional musicians listened to the audio samples categorizing them subjectively in 6 terms related to sonority, which were brilliant, opaque, translucent, solid, soft, and metallic.

From the FFT and DPE, it was possible to quantify the number of harmonics present for a given fundamental frequency, proposing a coefficient defined as the weighted average of the 
tones and intensities that expresses the magnitude of the harmonics present concerning the reference frequency or fundamental frequency. This indicator allowed to establish comparisons between sonorities or colors for each instrument, being the common factor in this family the associated with "soft" and "opaque" whose coefficients were 2.8 and 2.2 respectively. A peculiarity of these instruments is that, depending on the octave, the coefficient related to timbre changes by a factor of 3.5. This change gives greater versatility in sonorities for the instruments of this family.

From the spectrogram, the stages of sound evolution were analyzed. Each stage contributes to obtaining a defined sonority in a maximum time of 2 seconds for each sample, concluding that the attack and sustenance with which define the type of sound for each instrument to be listened to by professional musicians.

Finally, it is proposed to extend the method to other families of musical instruments for its generalization and potential application in the musical creation by sonorities as a compositional tool.

Acknowledgements: "This study was financed in part by the Coordenação de Aperfeiçoamento de Pessoal de Nível Superior - Brasil (CAPES)

\section{References}

[1] Strasburger, H., and Parncutt, H. Applying psychoacoustics in composition: Harmonic progressions of non-harmonic sonorities. Perspectives of new music,32(1), pages 88-129. 1994.

[2] Aramaki, M., Besson, M., Kronland-Martinet, R., \& Ystad, S. Timbre perception of sounds from impacted materials: behavioral, electrophysiological and acoustic approaches. In International Symposium on Computer Music Modeling and Retrieval, pages 1-17, Springer, Berlin, Heidelberg, 2008.

[3] Navalón Salas, I. et al. Spectral analysis of the sound obtained with oboe tudels made with different materials. Editorial Spanish Acoustic
Society, TECNIACÚSTICA 2015, 46 Spanish Acoustics Congress. 9th Iberian Acoustic Meeting. European Symposium on Virtual Acoustics and Ambisonics, Valencia, 2015.

[4] Schubert, E., Wolfe, J., and Tarnopolsky, A. Spectral centroid and timbre in complex, multiple instrumental textures. In Proceedings of the international conference on music perception and cognition, North Western University, Illinois, pages 112-116. 2004.

[5] González, Y. Study and characterization of the timbre quality associated with wooden wind instruments. 48th Spanish Acoustics Congress; Iberian Acoustic Encounter; European Symposium on Underwater Acoustics Applications; European Symposium on Sustainable Building Acoustics: A Coruña 3-6, pages 1138-1146. Spanish Acoustic Society.2017. 